\title{
Nanocrystal Skins with Exciton Funneling for Photosensing
}

\author{
Shahab Akhavan, Ahmet Fatih Cihan, Berkay Bozok, and Hilmi Volkan Demir*
}

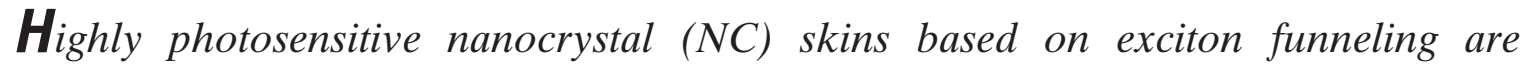
proposed and demonstrated using a graded bandgap profile across which no external bias is applied in operation for light-sensing. Four types of gradient NC skin devices (GNS) made of NC monolayers of distinct sizes with photovoltage readout are fabricated and comparatively studied. In all structures, polyelectrolyte polymers separating CdTe NC monolayers set the interparticle distances between the monolayers of ligand-free NCs to $<1 \mathrm{~nm}$. In this photosensitive GNS platform, excitons funnel along the gradually decreasing bandgap gradient of cascaded NC monolayers, and are finally captured by the NC monolayer with the smallest bandgap interfacing the metal electrode. Time-resolved measurements of the cascaded NC skins are conducted at the donor and acceptor wavelengths, and the exciton transfer process is confirmed in these active structures. These findings are expected to enable large-area GNS-based photosensing with highly efficient full-spectrum conversion.
\end{abstract}

\section{Introduction}

Solution-processed semiconductor nanocrystals (NCs) are of increasing interest for use in optoelectronic devices and offer advantages including low cost, flexibility, large device area, and spectral tunability based on the quantum size effect. $^{[1,2]}$ In contrast to conventional epitaxial growth of lattice-matched semiconductors, such solution-processed optoelectronic materials provide promising convenient inte-

\footnotetext{
S. Akhavan, A. F. Cihan, B. Bozok, Prof. Dr. H. V. Demir UNAM-Institute of Materials Science and Nanotechnology Department of Electrical and Electronics Engineering and Department of Physics Bilkent University Ankara 0680o, Turkey E-mail: volkan@stanfordalumni.org

Prof. Dr. H. V. Demir

School of Electrical and Electronic Engineering and School of Physical and Mathematical Sciences Nanyang Technological University

Singapore 639798, Singapore

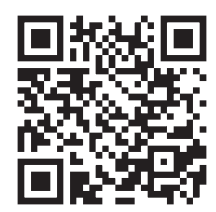

DOI: $10.1002 / \mathrm{smll} .201303808$ gration atop electronic substrates (e.g., using dip coating, spin coating and drop casting), where lattice mismatch problems do not arise. Therefore, these NCs are promising candidates for applications in diverse areas, such as in light-emitting diodes, ${ }^{[3]}$ solar cells, ${ }^{[4]}$ and photodetectors ${ }^{[5]}$ as well as for biolabeling ${ }^{[6]}$ and biosensing. ${ }^{[7]}$

Conventional photodetectors using NCs typically operate on the principle of collecting photogenerated charges, where an external bias must be applied for the charge collection. Although photodetectors comprising a Shockley-Schottky barrier can in principle be operated without an applied bias (in the open circuit mode), this would commonly come at the cost of decreasing the sensitivity. Recent research efforts increasingly focus on NC photodetectors that show a remarkable level of sensitivity ${ }^{[8]}$ and short response times. ${ }^{[9]}$ These photodetectors do, however, rely on photocurrent collection and commonly exhibit high dark current, which results in high noise levels and limits the device's detection capabilities. ${ }^{[10]}$

As an alternative photosensor architecture, NC skins offer the potential of high sensitivity for large-area applications because they operate on the principle of photogenerated voltage buildup in the absence of an external bias. With a single NC layer in the device structure, noise generation is significantly reduced, which enhances the device sensitivity. ${ }^{[11]}$ 
However, in this case, limiting these devices to a single NC layer for absorbing incident light hinders performance. In operation, with exciton disassociation due to the difference between the work functions of the NCs and the metal contact, electrons are trapped inside the NCs and holes are accumulated in the metal electrode, leading to a potential buildup across the device. Thus, although increasing the NC layer's thickness results in increased optical absorption, in the absence of any applied bias across the device, photogenerated excitons may not dissociate and sufficient charge may not accumulate. Thus, photosensitive NC skin performance depends not only on the optical absorption but also on the capacity to accumulate dissociated excitons.

In previous work, we reported that the highest possible sensitivity value for photosensitive NC skins is $104 \mathrm{mV}$ under the illumination intensity of $0.175 \mathrm{~mW} / \mathrm{cm}^{2}$ at a $350 \mathrm{~nm}$ wavelength, and that their operating wavelength range is limited to $350-450 \mathrm{~nm} \cdot{ }^{[12]}$ Other recent work demonstrated that enhancing $\mathrm{NC}$ film absorption via plasmonic nanoparticles increases NC skin sensitivity. ${ }^{[13]}$ However, this enhancement factor and the resulting operating wavelength range strongly depend on the limited localized plasmonic resonance band.

For the above reasons, we posit that by relying on the direct relationship between increasing the number of trapped electrons inside the NCs and subsequently the accumulated holes in the metal contact, a larger potential buildup in the photosensitive NCs skins will occur. We therefore pursue a cascaded structure of semiconductor NCs with the necessary bandgap gradient to enable exciton funneling, expecting a considerable improvement in the sensitivity. Here we fabricate photosensitive NC skins based on the Schottky barrier at the interface between the top CdTe NCs and Al contact (Figure 1a). A thin film of transparent indium tin oxide (ITO) underneath a dielectric film forms the opposite contact. Due to the device architecture and full surface coverage of the current blocking dielectric film made of $100 \mathrm{~nm}$ hafnium dioxide $\left(\mathrm{HfO}_{2}\right)$ and four bilayers of polydiallyldimethylammonium chloride-polysodium 4-styrenesulfonate (PDDA-PSS), the photosensitive $\mathrm{NC}$ skin itself acts as a capacitor. During fabrication, the NC monolayers are dip-coated with a computerized system on top of $\mathrm{HfO}_{2}$ film. Synthesis of CdTe NCs and monolayer assembly procedures are all explained in detail in our previous works as well as in the experimental section here. ${ }^{[12,13]}$

Figure 1a shows a schematic drawing of the closely packed NC monolayers. Because of the considerable change in the NC surface coverage as the number of polyelectrolyte bilayers changes, ${ }^{[14]}$ we found that four bilayers of PDDAPSS were necessary to provide a uniform film with enough surface coverage for depositing the CdTe NC monolayer. In operation, light incident through the glass substrate generates excitons in the NC monolayers. The excitons funnel along the gradually decreasing bandgap gradient of cascaded $\mathrm{NC}$ monolayers, and are finally captured by the NC monolayer with the largest bandgap adjacent to Al contact, which acts as a hole acceptor. These excitons are then dissociated at the $\mathrm{NC}-\mathrm{Al}$ interface, and thus, holes migrate to the $\mathrm{Al}$ contact due to the HOMO/LUMO band alignment of the NC layer and the Al workfunction, as shown in Figure 1b. ${ }^{[15]}$ Electrons, however, remain trapped inside the NCs.

Under illumination, photovoltage buildup reaches a peak at which it is equal to the RC decay rate of the whole circuitry and at which the external circuitry inclines to neutralize the trapped charges in the NCs. After reaching this peak, and with the incident light off, the net potential diminishes and even becomes negative due to the potential of the trapped electrons. In consequence, the negative potential reaches its lowest value and returns to zero as the trapped charges escape over time. The amount of time for the net potential value to return to zero demonstrates the effect of the trapped electrons and is related to the time needed to deplete these charges. As a whole, light-sensitive skins can be considered as an open-circuit photosensor that rely on photovoltage buildup. As a matter of fact, this device can work most efficiently in this mode because of its high resistivity.

Controlling NC surface passivation is critical for efficient device operation, and because no external bias is applied through our cascaded NC structure, efficient exciton transfer must be achieved through some other method. NCs are normally separated from each other via ligands of isolating organic molecules that passivate NC surfaces through oxidation and chemical modifications. The ligands also control electronic properties of NCs including conductivity and (a)

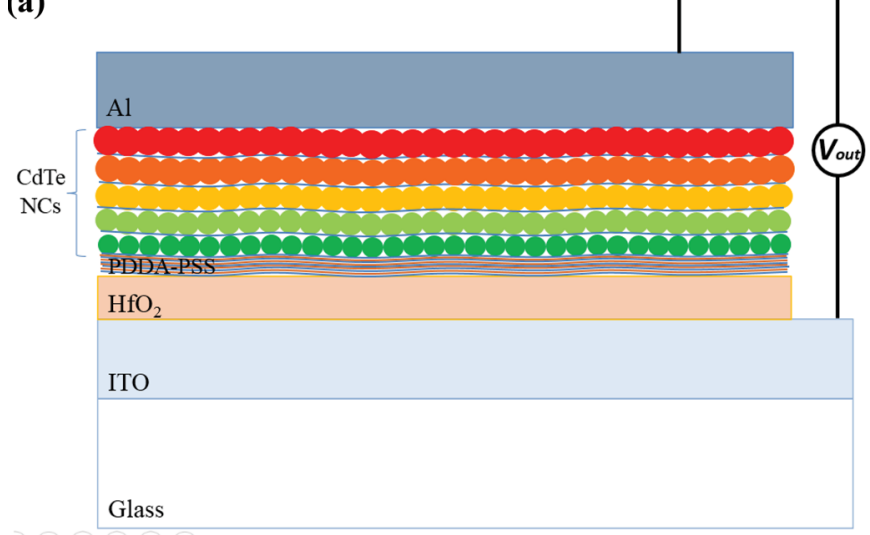

(b)

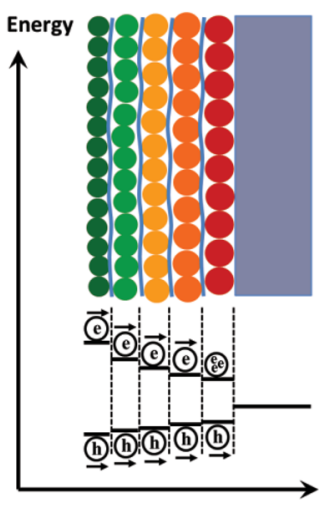

Figure 1. (a) Schematic of the photosensitive NC skins, consisting of closely packed layers of ligand-free CdTe NCs. (b) Alignment of the conduction and valence bands of the CdTe NC layers and the workfunction of the Al contact. Excitons transfer through the layers from smaller to larger NCs because of the bandgap gradient. 
charge mobility. ${ }^{[16,17]}$ To bring NCs closer, we removed the ligands by adding isopropanol to the CdTe NC solution and centrifuging the mixture (Figure S1).

For this work, we synthesized water-soluble CdTe NCcapped thioglycolic acid (TGA) ligands of different sizes according to Rogach et al., and calculated the NC diameters from their extinction spectra. ${ }^{[18,19]}$ These diameters were 2.90, $2.98,3.19,3.62$, and $3.71 \mathrm{~nm}$, leading to the first excitonic peak wavelengths of 525, 532, 545, 595, and $605 \mathrm{~nm}$, respectively (Figure S2).

\section{Results and Discussion}

In this work we demonstrate that with the proper energy gradient of NC skins composed of several monolayers, photosensitivity can be substantially enhanced and extremely broadband photosensitivity can be achieved. Figure 2 shows the photovoltage buildup as a function of time across four devices that we fabricated. For the device utilizing a single NC layer as the photoactive layer, we show that varying photovoltage buildup amounts at different excitation wavelengths (Figure 2a). Increasing the number of the same NC layers from one to five leads to greatly enhanced absorption, but we observe voltage buildup suppression and a consequent reduced level of the device sensitivity compared to the single layer. Voltage buildup suppression can be explained by the self-absorption effect in the lowermost NC layers; fewer fractions of photons reach the topmost NC layers, and excitons photogenerated in the bottom NC layers cannot make it to the top, which leads to fewer electrons and holes being trapped in the NCs and stored at the Al contact. Additionally, in the absence of a bandgap gradient, each exciton tends to remain in the layer where it was created until it is recombined. Therefore, given that the device performance efficiency is strongly dependent on the fraction of photons absorbed, favorable thickness must be used for the photoactive layer.

Previously Franzl et al. proposed that NC funnel-like bandgap profiles transfer excitons to the largest NCs with the smallest bandgap. ${ }^{[20]}$ The funnel structure recycles the trapped and lost electron-hole pairs, leading to enhanced photoluminescence (PL) of the largest NCs in optically pumped structures. ${ }^{[21,22]}$ Most recently, utilizing graded bandgap profile in photovoltaic devices has also gained a strong interest and enhanced the fill factor of solar cells. ${ }^{[23]}$ Here, different than the previous reports, we demonstrate the first account of exciton funneling in photosensitive $\mathrm{NC}$ platform for light-sensing and systematically study the energy transfer in these active devices. In this work, we focus on three structures composed of five NC monolayers, where the NC sizes differ depending on the structure (see Figure 2b-d). The bandgaps of the CdTe NCs are adjusted to transfer photogenerated excitons from the layers comprising smaller NCs to the layer comprising the largest NCs, which is immediately

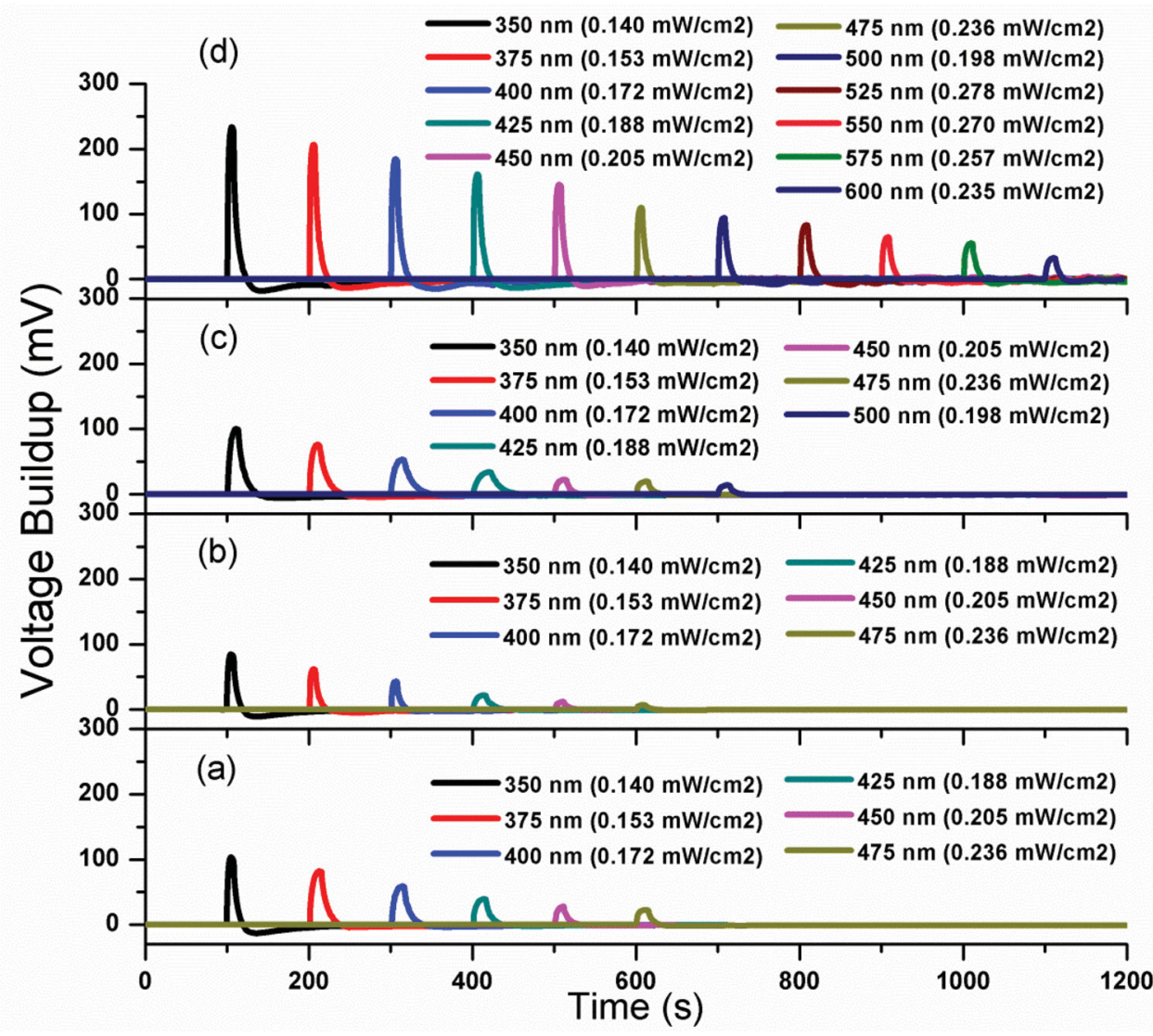

Figure 2. Experimental photovoltage buildup results of photosensitive NC skins at different excitation wavelengths and intensity levels based on (a) a single layer of NCs, (b) five layers of NCs of the identical size, (c) five layers of NCs ranging from large to small, and (d) five layers of NCs ranging from small to large. All photovoltage buildup results already reached steady-state before the light was turned off. 


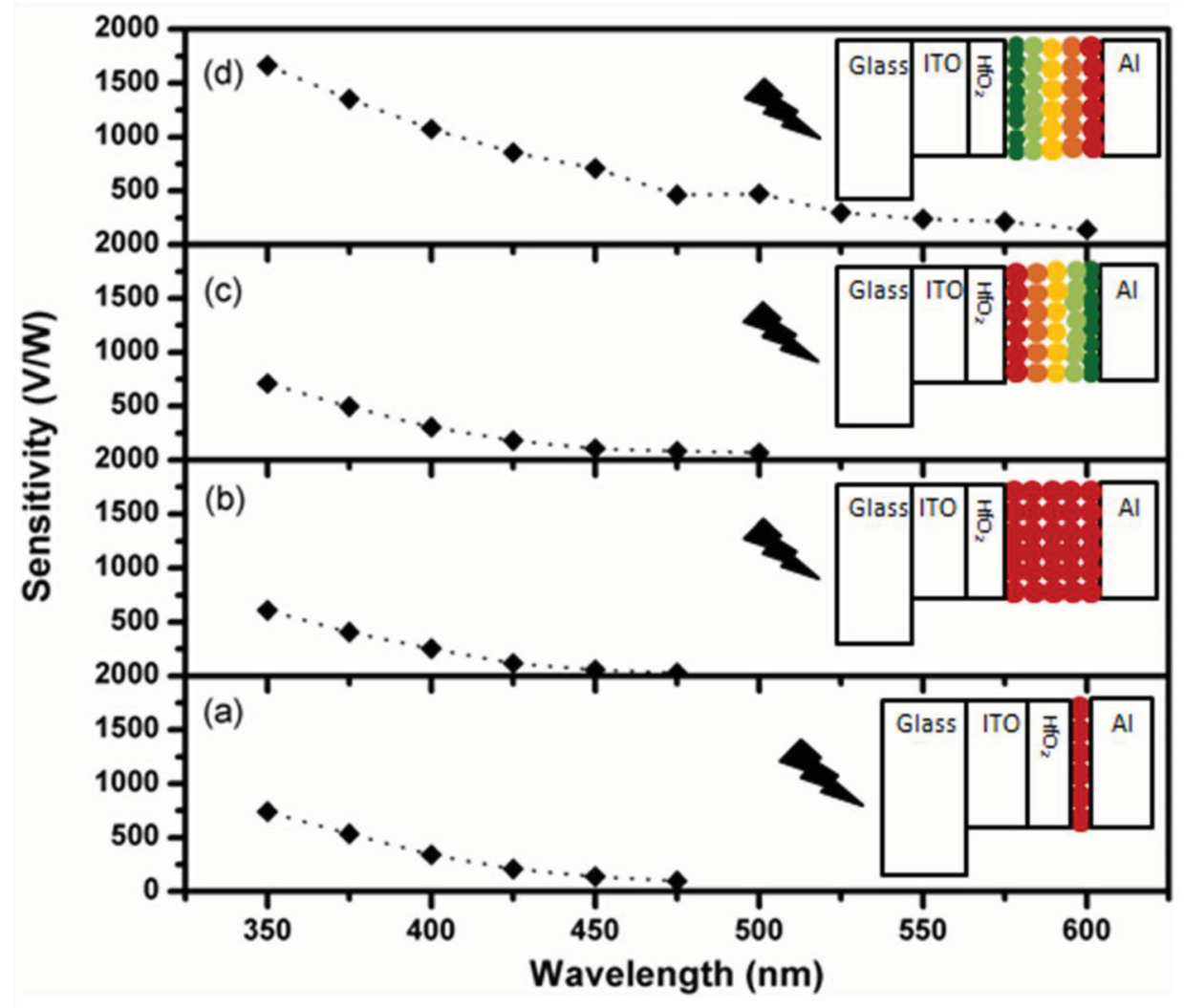

Figure 3. A comparison of the corresponding sensitivities as a function of excitation wavelength based on (a) a single layer of NCs, (b) five layers of NCs of the identical size, (c) five layers of NCs ranging from large to small, and (d) five layers of NCs ranging from small to large. Inset figures present the device schematics and the direction of incident light.

underneath the $\mathrm{Al}$ contact. Compared to the device with a single NC layer, the device with multiple NC layers placed within the proper bandgap gradient exhibits a significant enhancement in the sensitivity and a considerable extension in the operating wavelength range (see Figure $2 \mathrm{a}, \mathrm{d}$ ).

The observed enhancement in the proposed cascaded structure's voltage buildup behavior can be attributed to the excitonic transfer from the smallest NCs to the largest NCs. After the excitons reach the largest $\mathrm{NC}$ layer, the excitons dissociate and the holes migrate and accumulate at the $\mathrm{Al}$ contact. Similarly, the electrons are trapped inside the NCs, as observed from the negative voltage levels of the photovoltage spectrum (Figure 2).

As evident from Figure 2d, we achieve a more than twofold enhancement in photovoltage buildup in the multilayer device compared to the monolayer device. Furthermore, the cascaded NC skin structure exhibits considerably improved spectral behavior, with its operating wavelength range between 350 and $600 \mathrm{~nm}$.

To investigate the effect of the bandgap gradient in a funnel-like device, we study the order dependence of $\mathrm{NC}$ layers by fabricating a converse structure (accumulating photogenerated excitons from layers with smaller NCs to layers with larger NCs, now placed far away from the Al contact), whose voltage buildup is presented in Figure 2c. The low performance of the converse funnel-like device is attributed to the depopulation of photogenerated excitons from the layers with smaller NCs because of the energy gradient in the reverse direction. Thus, fewer numbers of holes accumulate at the $\mathrm{Al}$ contact and consequently, fewer electrons become trapped in the NC layer under the $\mathrm{Al}$ contact. This observation strongly coincides with the proposed excitonic transfer process of $\mathrm{NC}$ exciton population and depopulation dynamics via nonradiative energy transfer toward the layer with the largest NCs.

As can be seen from Figure 2c,d the response time of the converse funnel-like device is slower than the gradient device with the ideal bandgap. This result indicates that, although the excitons become disassociated in the former device, due to the unfavorable bandgap gradient, they separate from the Al contact and remain longer in the device, which results in a slower operation.

We calculated $\mathrm{NC}$ skin photosensitivity according to the ratio of $\mathrm{S}=\mathrm{V} / \mathrm{P}$, where $\mathrm{V}$ is the photovoltage buildup and $\mathrm{P}$ is the incident optical power impinging on the device. Figure 3 demonstrates the corresponding sensitivity curves as a function of the excitation wavelength. Similar to the observed photovoltage buildup behavior, the sensitivity spectra also follow the $\mathrm{NC}$ absorption spectrum and increase as the photon energy increases. Additionally, we also present the quantum efficiency of photosensitive NC skins in terms of voltage buildup per absorbed photon (in units of $\mathrm{V}$ per photon) in Figure S3.

The absorption and emission spectra of the distinct sizes of the CdTe NCs show considerable overlap; therefore, as the physical basis for exciton transfer, nonradiative exciton 
transfer (NRET) from small NCs to large NCs can be considered to be a very likely process. ${ }^{[24-26]}$ To further verify the presence of NRET between these NC layers, we conducted time-correlated single-photon counting experiments (Picoquant, Fluotime 200) with a pulsed excitation laser diode at $375 \mathrm{~nm}$ having a calibrated time resolution of $32 \mathrm{ps}$. We conducted time-resolved fluorescence (TRF) measurements of the cascaded NC structure at the emission peaks of the smallest (donor-side) and largest (acceptor-side) NCs, which were 545 and $645 \mathrm{~nm}$, respectively. These peak wavelengths correspond to the respective emission peaks of the dark green layer (smallest NCs) and the red layer (largest NCs). The dynamics obtained in the experiments include the fluorescence decays of the NCs convolved with the system instrument response function (IRF) with a pulse width of $200 \mathrm{ps}$. When analyzing the TRF results, we used multiexponential least chi-square fittings in the reconvolution mode (Picoquant Fluofit).

To verify the existence of NRET, we conducted experiments on five monolayers of donor-only NCs, acceptor-only NCs, and cascaded samples with the bandgap gradient. As evident from Figure 4, there is a clear difference between the TRF decays of the donor-only sample and the hybrid sample for the measurements at $545 \mathrm{~nm}$, the PL peak of the donor NCs. The amplitude-averaged donor lifetime becomes $0.304 \mathrm{~ns}$ in the hybrid sample and $0.567 \mathrm{~ns}$ for the donoronly sample, which is a clear indicator for the presence of the NRET process from the layer with the smallest NCs to the four layers with larger NCs. To verify exciton feeding to the layer with the largest NCs (acceptor NCs) from the four layers with the smaller NCs, we took TRF measurements of the acceptor-only sample and the hybrid sample at the emission peak $(645 \mathrm{~nm})$ of the red (largest) NCs. The acceptorside average lifetime of the hybrid sample was $2.86 \mathrm{~ns}$,

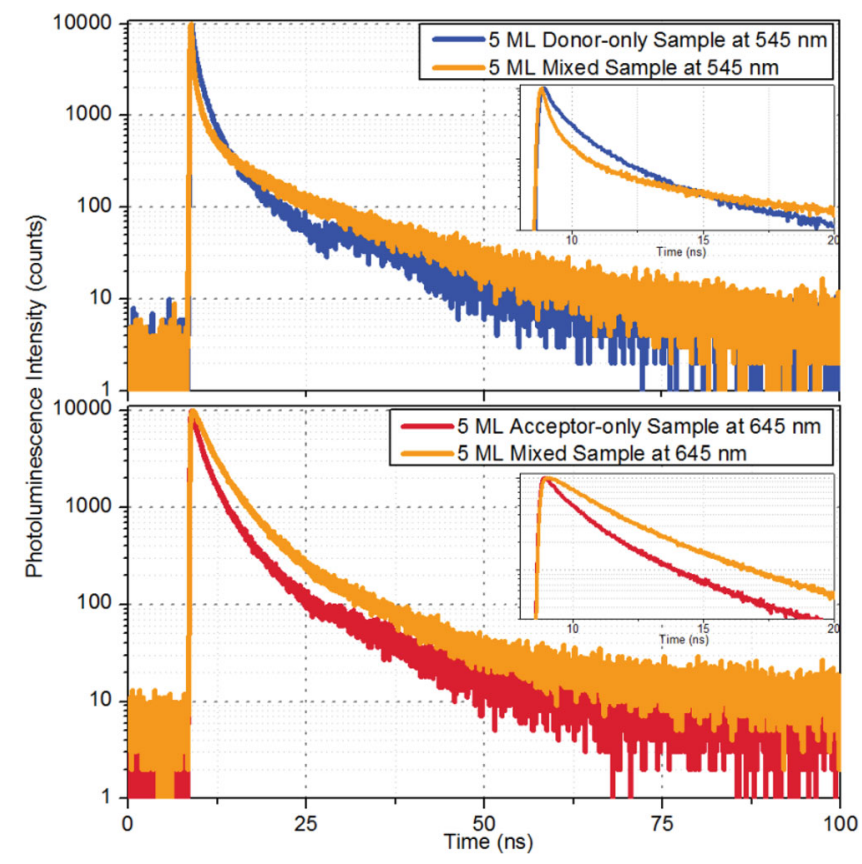

Figure 4. Time-resolved PL decays of the five donor NC layer samples, the five acceptor NC layer samples, and the five hybrid-layer samples. Insets show the first 20 ns parts of the decays. whereas the acceptor-only sample lifetime was 1.66 ns. These significant decay lifetime differences are shown in Figure 4, and the PL spectra are shown in Figure S4. Furthermore, the prolonged acceptors feeding time (over some nanoseconds) can be attributed to the transfer of trapped excitons in the smaller NCs and their transfer in stepwise manner to the largest NCs. ${ }^{[27]}$ These observations indicate that NRET occurs between these NC layers, and therefore, we can attribute the enhancement in photovoltage buildup and the consequent sensitivity improvement to NRET towards the top NC layer with the desirable energy gradient of the $\mathrm{NC}$ films.

Although the NRET from the small NCs to the large ones is evident, its efficiency could in principle be higher. The ligand removal process may cause new nonradiative decay channels in the NCs, thus resulting in very fast NC decay; hence, NRET may become less effective. It is important to note that the monolayer-based device can build up approximately $100 \mathrm{mV}$ under $0.170 \mathrm{~mW} / \mathrm{cm}^{2}$ at $350 \mathrm{~nm}$. The cascaded monolayer device in the proper funnel structure was thus expected to show a photovoltage buildup of about $500 \mathrm{mV}$ under similar experimental conditions, but the observed voltage buildup was only about $245 \mathrm{mV}$ in all our fabricated funnel-based devices. This deficiency in performance indicates that exciton transfer in the funnel structure is not fully efficient. Excitons can be trapped between NC layers because of intervening polyelectrolyte layers, surface states, and defects created in the layer-by-layer assembly, and we surmise that such events occurred in these experiments.

\section{Conclusion}

Photosensitive NC skins utilizing a bandgap gradient of cascaded NC layers demonstrate significant sensitivity enhancement and extension in their operating wavelength range. This performance improvement is attributed to exciton transfer from the layer with the smallest NCs to the layer with the largest NCs. In this work, by conducting TRF measurements of NC structures, we observe and attribute the reduction in average PL lifetime of the smallest NCs and the increase in PL lifetime of the largest NCs compared to the lifetimes of the same NCs in the hybrid sample to exciton transfer through the NC layers. These observations strongly coincide with the device's sensitivity enhancement and operating wavelength range extension. The cascaded NC structure proposed here offers important implications for future designs of photosensing NC devices, which have the potential to be semi-transparent and/or flexible. These results are expected to enable engineering large-area NC-based photosensitive platforms, including smart windows and facades.

\section{Experimental Section}

Device Fabrication: ITO film deposited on a glass substrate was washed in a mixture of $2 \mathrm{~mL}$ Hellmanex in $100 \mathrm{~mL}$ Milli-Q water for 20 min using ultrasonication, followed by $20 \mathrm{~min}$ in water, $20 \mathrm{~min}$ in acetone and $20 \mathrm{~min}$ in isoproponal. After that, $100 \mathrm{~nm}$ 
thick $\mathrm{HfO}_{2}$ dielectric film was deposited using ALD. Subsequently, in order to deposit NCs, we used layer-by-layer self-assembly with a computerized system. Lastly, a $100 \mathrm{~nm}$ Al contact layer was laid down on top of NCs monolayers via a thermal evaporator.

Device Characterizations: To characterize the photosensitive NC skins, we used Agilent Technologies B1500A Semiconductor Parameter Analyzer and a Xenon light source, with an integrated monochromator as the excitation source. We measured optical power with a Newport optical power meter (1835C). During all measurements, the devices were grounded from the ITO side and connected with a load resistance of $200 \mathrm{M} \Omega$.

Time-Resolved Fluorescence Measurements: We used a pulsed laser excitation $(3.3 \mathrm{eV})$ with a pulse width of less than $50 \mathrm{ps,}$ focused to a spot size of $37 \mu \mathrm{m}^{2}$ on the sample and at a repetition rate of $5 \mathrm{MHz}$. In the experiments, the excitation fluence was around $5 \times 10^{12}$ photons $/ \mathrm{cm}^{2}$ per pulse which is low enough for the single exciton regime operation. The TRF decays were fitted with 4-exponentials, which led to the best $\chi^{2}$ values. In all of these fittings, $\chi^{2}$ value was between 0.9 and 1.1. The amplitude averaged lifetime value is calculated via $\tau_{\text {avg }}=\left(\Sigma\left(A_{i} \tau_{i}\right)\right) /\left(\Sigma\left(A_{i}\right)\right)$.

Layer-by-Layer Assembly: The TGA ligands were removed from the NC surfaces by adding isopropanol into the CdTe NC solution and centrifuging the mixture for three times. During the layer-bylayer film assembly, NC solution was rigorously stirred in order to prevent the precipitation. The layered samples were prepared through alternating deposition of the positively charged PDDA (2 $\mathrm{mg} / \mathrm{mL}$ in $0.1 \mathrm{M} \mathrm{NaCl}$ solution) and the negatively charged PSS (2 $\mathrm{mg} / \mathrm{mL}$ in $0.1 \mathrm{M} \mathrm{NaCl}$ solution). Dipping time of $5 \mathrm{~min}$ in each solution was chosen, and samples were rinsed with Milli- $Q$ water for $1 \mathrm{~min}$ after each step. Four alternating bilayers of strongly positive and negative polyelectrolyte polymers were deposited on the $\mathrm{HfO}_{2}$ layer. On top of those, a monolayer of negatively charged CdTe NCs was deposited, followed by a final positive polyelectrolyte monolayer. In the samples consisting of five monolayers of different-sized CdTe NCs, monolayers were also separated with PDDA. For NC monolayer formation, the samples were dipped into the NC dispersion of $\mathrm{CdTe}$ for $20 \mathrm{~min}$ and rinsed with water again for 1 min. All samples were prepared and kept in darkness to minimize NC photodegradation.

Synthesis of Nanocrystals: $4.59 \mathrm{~g}$ of $\mathrm{Cd}\left(\mathrm{ClO}_{4}\right)_{2} \cdot 6 \mathrm{H}_{2} \mathrm{O}$ was dissolved in $500 \mathrm{~mL}$ of Milli-Q water followed by addition of $1.33 \mathrm{~g}$ TGA and adjustment of the $\mathrm{pH}$ to $11.8-12.0$. Then, we conducted $\mathrm{H}_{2}$ Te gas flow by reacting $0.8 \mathrm{~g} \mathrm{Al}_{2} \mathrm{Te}_{3}$ with $\mathrm{H}_{2} \mathrm{SO}_{4}$ with a slow $\mathrm{Ar}$ flow. At $100^{\circ} \mathrm{C}$, the nucleation and growth of the NCs was initiated.

\section{Supporting Information}

Supporting Information is available from the Wiley Online Library or from the author.

\section{Acknowledgements}

We gratefully acknowledge financial support by ESF EURYI, EU FP7 Nanophotonics4Energy NoE; by TUBITAK under Project Nos. EEEAG
110E217, 111E186, and 112E183; and by NRF-CRP6-2010-02 and NRF RF 2009-09. HVD acknowledges additional support from TUBA.

[1] S. V. Gaponenko, Introduction to Nanophotonics Cambridge University Press, NY, USA 2010.

[2] T. Erdem, H. V. Demir, Nat. Photonics 2011, 5, 126.

[3] T. Erdem, H. V. Demir, Nanophotonics 2013, 2, 57-81.

[4] X. Lan, J. Bai, S. Masala, S. M. Thon, Y. Ren, I. J. Kramer, S. Hoogland, A. Simchi, G. I. Koleilat, D. Paz-Soldan, Z. Ning, A. J. Labelle, J. Kim, G. Jabbour, E. H. Sargent, Adv. Mater. 2013, 25, 1769-1773.

[5] G. Konstantatos, M. Badioli, L. Gaudreau, J. Osmond, M. Bernechea, F. P. G. de Arquer, F. Gatti, F. H. L. Koppens, Nat. Nanotechnol. 2012, 7, 363-368.

[6] X. Michalet, F. F. Pinaud, L. A. Bentolila, J. M. Tsay, S. Doose, J. J. Li, G. Sundaresan, A. M. Wu, S. S. Gambhir, S. Weiss, Science 2005, 307, 538-544.

[7] P. Alivisatos, Nat. Biotechnol. 2004, 22, 47-52.

[8] G. Konstantatos, I. Howard, A. Fischer, S. Hoogland, J. Clifford, E. Klem, L. Levina, E. H. Sargent, Nature 2006, 442, 180-183.

[9] F. Prins, M. Buscema, J. S. Seldenthuis, S. Etaki, G. Buchs, M. Barkelid, V. Zwiller, Y. Gao, A. J. Houtepen, L. D. A. Siebbeles, H. S. J. van der Zant, Nano Lett. 2012, 12, 5740-5743.

[10] J. P. Clifford, G. Konstantatos, K. W. Johnston, S. Hoogland, L. Levina, E. H. Sargent, Nat. Nanotechnol. 2009, 4, 40-44.

[11] E. L. Dereniak, G. D. Boreman, Infrared Detectors and SystemsWiley \& Sons, NY, USA 1996.

[12] S. Akhavan, B. Guzelturk, V. K. Sharma, H. V. Demir, Opt. Exp. 2012, 20, 25255-25266.

[13] S. Akhavan, K. Gungor, E. Mutlugun, H. V. Demir, Nanotechnology 2013, 24, 155201.

[14] J. W. Ostrander, A. A. Mamedov, N. A. Kotov, J. Am. Chem. Soc. 2001, 123, 1101-1110.

[15] J. Jasieniak, M. Califano, S. E. Watkins, ACS Nano 2011, 5, 5888-5902.

[16] J. M. Luther, M. Law, Q. Song, C. L. Perkins, M. C. Beard, A. J. Nozik, ACS Nano 2008, 2, 271-280.

[17] M. V. Jarosz, V. J. Porter, B. R. Fisher, M. A. Kastner, M. G. Bawendi, Phys. Rev. B 2004, 70, 195327.

[18] A. L. Rogach, T. Franzl, T. A. Klar, J. Feldmann, N. Gaponik, V. Lesnyak, A. Shavel, A. Eychmüller, Y. P. Rakovich, J. F. Donegan, J. Phys. Chem. C 2007, 111, 14628-14637.

[19] P. Reiss, M. Protière, L. Li, Small 2009, 5, 154-168.

[20] T. Franzl, T. A. Klar, S. Schietinger, A. L. Rogach, J. Feldmann, Nano Lett. 2004, 4, 1599-1603.

[21] T. A. Klar, T. Franzl, A. L. Rogach, J. Feldmann, Adv. Mater. 2005, $17,769-773$.

[22] F. Xu, X. Ma, C. R. Haughn, J. Benavides, M. F. Doty, S. G. Cloutier, ACS Nano 2011, 5, 9950-9957.

[23] I. J. Kramer, L. Levina, R. Debnath, D. Zhitomirsky, E. H. Sargent, Nano Lett. 2011, 11, 3701-3706.

[24] S. Nizamoglu, P. L. Hernandez-Martinez, E. Mutlugun, D. U. Karatay, H. V. Demir, Appl. Phys. Lett. 2012, 100, 241109.

[25] C. R. Kagan, C. B. Murray, M. G. Bawendi, Phys. Rev. B 1996, 54, 8633-8643.

[26] S. A. Crooke, J. A. Hollingsworth, S. Tretiak, V. I. Klimov, Phys. Rev. Lett. 2002, 89, 186802.

[27] S. Mayilo, J. Hilhorst, A. S. Susha, C. Hohl, T. Franzl, T. A. Klar, A. L. Rogach, J. Feldmann, J. Phys. Chem. C 2008, 112, 14589-14594.

Received: December 12, 2013

Revised: January 22, 2014

Published online: March 5, 2014 